\title{
Jet development and impact load of underwater explosion bubble on solid wall
}

\author{
Zhao-Li Tianª,b, Yun-Long Liu ${ }^{\mathrm{a}, \mathrm{b}, *}$, A-Man Zhang ${ }^{\mathrm{a}, *}$, Longbin $\mathrm{Tao}^{\mathrm{c}}$, Liang \\ Chen $^{\mathrm{d}}$ \\ ${ }^{a}$ Harbin Engineering University, Harbin, China \\ ${ }^{b}$ Brown University, Providence, USA \\ ${ }^{c}$ Department of Naval Architecture, Ocean 83 Marine Engineering, University of \\ Strathclyde, Glasgow, United Kingdom \\ ${ }^{d}$ China Shipbuilding Industry South Corporation, Shenzhen, China
}

\begin{abstract}
The damage effects of an underwater explosion have always been a crucial problem in the ship mechanics. Notably, the bubble evolution and the jet impact load are one of the most difficult parts in the shock-resistance design of ship structures due to the discontinuities and significant nonlinear deformation. In this paper, the Eulerian finite-element method is introduced to continuously simulate the shock wave and non-spherical bubble evolution stages, and to evaluate the explosion impact load on a nearby solid wall with the volume of fluid method and pressure balance technique used to solve the multi-phase flow. The numerical model is established in a cylindrical coordinate system and validated by comparing the results with a spark-generated bubble experiment. After that, based on the present model, the shock wave propagation and the bubble evolution are simulated to study the characteristics of the impact loads of an underwater explosion. Besides, the influences of the wall location (upside or downside) and the stand-off distance from the wall are also analyzed. The results show that the features of the jet impact load are much more complicated than those of the shock wave. Nearby a downside wall, the buoyancy and Bjerknes force compete to dominate the bubble motion with opposite influences. By contrast, They enhance the
\end{abstract}

\footnotetext{
*Corresponding authors

Email addresses: yunlong_liu@hrbeu.edu.cn (Yun-Long Liu), zhangaman@hrbeu.edu.cn (A-Man Zhang)
} 
effect of each other to develop a liquid jet towards the upside wall. The pressure peak, impact range, and duration time nonlinearly depend on the combination of the case parameters and are not monotonic to a single one. Within a proper range of the parameter combination, the jet impact load can reach its maximum and be more destructive than the shock wave because of a comparable pressure peak and a much longer duration.

Keywords: Bubble dynamics; Jet; Underwater explosion; Impact load

\section{Introduction}

Underwater explosion load is not only the main threat to the survival of warships (Cole, 1948; Klaseboer et al., 2005a; Liang and Tai, 2006; Liu et al., 2018a; Tian et al., 2019), but also is one of the critical values for the design of marine structures. The underwater explosion consists of complicated physical and chemical processes, and its detailed features vary with the surrounding environment. The underwater explosion process is usually divided into shock wave phase whose load form is shock wave and bubble phase containing bubble pulsation and jet development. Because there are large dimension ratios in both time and space between these two phases, it is hard to find a numerical method to balance the accuracy and efficiency. Thus, the simulations of these two phases were used to be treated separately.

During the shock wave phase, the load is usually calculated by the linearized acoustic method (Lai and Khan, 2011; Sprague and Geers, 2004) or by the semi-experimental expressions concluded by the empirical data in engineering applications (Cole, 1948), which is valid for the mid-far field explosion while the precision decreases along with the increase of the nonlinearity in the near field cases. As for the bubble phase, the incompressible flow assumption is widespread in the numerical simulation, because of the absence of sound speed which severely restricts the time increment. It is found that the bubble produces a jet under the effects of buoyancy and surrounding boundaries (Plesset and Chapman, 1970; Lenoir, 1976) and that the jet form varies along with their non-linear combination (Plesset and Chapman, 1970; Hooton et al., 1994; Zhang et al., 2006; Blake and Gibson, 1987; Lee et al., 2007; Cui et al., 2018; Li et al., 2019).

Explosion load can threaten the nearby structures, no matter with shock wave load or the bubble load. An increasing number of researchers carried out related works on bubble dynamics and its impact load. Among 
the various numerical methods in bubble dynamics, the boundary element method (BEM) has been one of the most salient methods. Khoo (Zhang et al., 2015; Lee et al., 2007; Wang et al., 2005, 2003; Dadvand et al., 2013; Klaseboer et al., 2005a), Wang (Wang, 2004; Wang et al., 2005; Wang, 2013), Zhang (Zhang et al., 2015; Zhang and Liu, 2015) and their collaborators have pushed forward the application of BEM in this field. However, these studies are premised on incompressible or weakly compressible field assumptions. For most time of a pulsation cycle of the underwater explosion bubble, the Mach number is small enough to ignore the compressibility. But when the initial shock wave or the jet impact lead to discontinuities, the BEMs cannot capture these features. Besides, the explicit interface tracking needs complicated unique numerical treatments and often leads to unstable simulations (Wang et al., 2003; Wang, 2013; Li et al., 2019; Zhang and Liu, 2015) because of the significant change of the mesh topology after the jet penetration.

There are also some researchers seeking new methods other than the BEM to study bubble dynamics. Johnsen and Colonius (2009) adopted the Finite Volume method (FVM) with high order WENO scheme to study the nonspherical bubble collapse induced by a shock wave. Liu et al. (2017) studied the bubble collapse based on the combination of front tracking method and FVM. Tian et al. (2018) and Liu et al. (2018b) numerically simulated the explosion from the shock wave phase to the bubble phase contiguously and analyzed the breaking and re-closure of a bubble with the Eulerian finiteelement method (EFEM). In Liu et al. (2019), the EFEM is modified to resolve the dynamics of an oscillating bubble initially at a liquid-liquid interface. Anwar (2019) employed the lattice Boltzmann method to study the rising bubbles interacting with cylindrical obstacles and coalescence.

In allusion to the problems mentioned above, we simulate and analyze the jet development and impact load of underwater explosion bubble on the solid wall based on the EFEM in this paper, taking full account of the compressibility of the flow fluid. The context is organized as flows. Firstly, considering the compressibility of the field and problem configuration, we combine the Eulerian and Lagrangian viewpoints and establish the EFEM using operator split technique, then construct the material interfaces with the Volume of Fluid (VOF) method and the pressure balance in the multiphase flow elements. Secondly, the model is validated by a spark-generated bubble experiment. Thirdly, we analyze the evolution of bubble jet and impact loads on the upside and downside solid wall for the shock wave and bubble phases. In the end, some conclusions are given based on the presented investigation. 


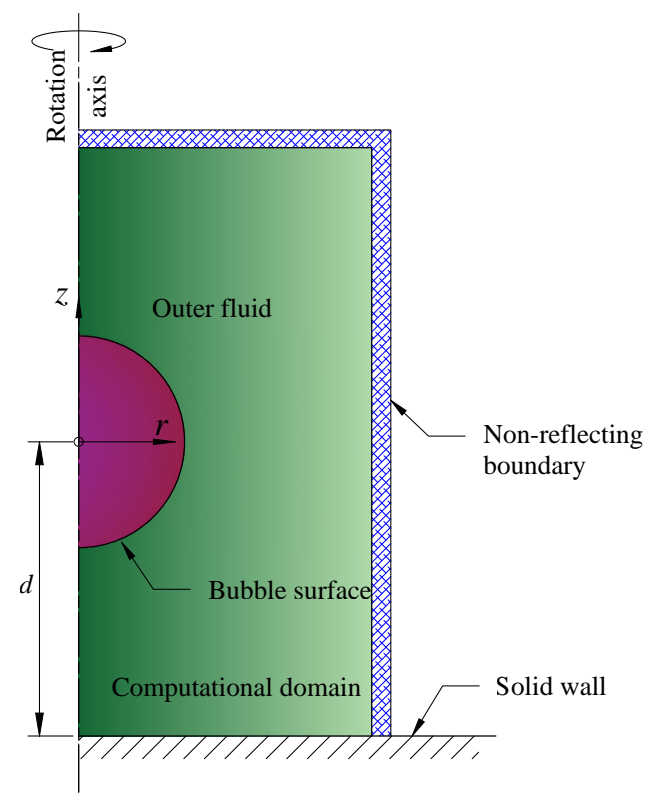

Figure 1: Configuration of the underwater explosion near a solid wall

\section{Theoretical and numerical models}

\subsection{Problem description and assumptions}

In this paper, the underwater explosion bubble dynamics near a solid horizontal wall is concerned, as shown in Fig.1. Following previous works in underwater explosion bubble dynamics (Zhang et al., 2015; Klaseboer et al., $2005 \mathrm{~b}, \mathrm{a})$, the detonation process of the explosive is treated as completed in an instant, i.e., the bubble is filled with high-pressure explosion products initially in still water. This assumption is reasonable for most explosives with a detonation wave speed faster than sound speed such that the surrounding water is undisturbed when the detonation wave reaches the material interface. All other boundaries are assumed to be far enough from the bubble except a planar solid wall located in the vicinity of the bubble. Because of the pressure difference between the bubble interface, an initial Riemann problem is proposed. Then, a shock wave propagates outward in the surrounding water while a rarefaction wave propagates inward in the internal gas. In the meantime, the bubble interface expands as the contact discontinuity. Without the influences of the solid wall and the buoyancy, the bubble will oscillate spherically until all the energy is dissipated through the emitted pressure 
waves. If the normal direction of the wall parallels to that of the gravity, the problem can be assumed to be axisymmetric. Thus, we adopt a cylindrical coordinate system whose origin locating at the initial bubble center and the $z$ axis pointing upward. The original distance between the bubble center and the solid wall is denoted as $d$. The impenetrable boundary condition is applied on the axis boundary and the solid wall. The non-reflecting boundary condition proposed by Liu et al. (2018b) is applied on the outer edge of the computational domain except the solid wall to eliminate the spurious reflections.

\subsection{Eulerian finite-element method}

In this paper, the EFEM with operator split is adopted to simulate the motion of the bubble with the compressibility of surrounding fluid considered. The main idea of this method is to split each time increment into two phases. In the first one, namely the Lagrangian phase, the computational mesh moves along with the fluid material. Thus, this phase is solved with a Lagrangian scheme. After that, the fluid material is assumed to be fixed at the new spatial position, and the mesh is moved back to the original place. In this phase which is so-called Eulerian phase, the convection of the fluid through all element interfaces are considered. Thus, at the end of the increment, the fluid material is advanced, but the mesh is still the same as that at the increment beginning.

As for the Lagrangian phase, following the previous studies on bubble dynamics, the surface tension effect, viscosity, and thermal conduction are all neglected (Klaseboer et al., 2005a; Liu et al., 2017, 2018b; Zhang and Liu, 2015). Then, the stress tensor is defined as the inverse of the pressure scalar $p$. The motion of the flow field can be described with the Euler equations:

$$
\begin{gathered}
\frac{\mathrm{d} \rho}{\mathrm{d} t}+\rho \nabla \cdot \mathbf{u}=0, \\
\rho \frac{\mathrm{d} \mathbf{u}}{\mathrm{d} t}+\nabla p-\rho \mathbf{g}=0, \text { and } \\
\frac{\mathrm{d} \rho e_{i n}}{\mathrm{~d} t}+p \nabla \cdot \mathbf{u}=0
\end{gathered}
$$

where $\rho$ is the density of the fluid; $\mathbf{u}=\left[u_{r}, u_{z}\right]$ is the velocity vector; $\mathbf{g}$ is gravitational acceleration; $e_{i n}$ denotes the specific internal energy per unit mass. The divergence of velocity in the coordinate system is defined as

$$
\nabla \cdot \mathbf{u}=\frac{\partial u_{r}}{\partial r}+\frac{\partial u_{z}}{\partial z}+\frac{1}{r} u_{r}
$$


The system above is solved with the framework of EFEM (Wu and $\mathrm{Gu}$, 2012) as described below. Denote $T_{h}$ as the non-overlapping partition on the computational domain $\Omega$ and $\mathbf{a}_{h} \in V_{h}$ is the numerical solution of the material acceleration $\mathrm{d} \mathbf{u} / \mathrm{d} t$, where $V_{h}$ is the finite-element space and is taken as piecewise bilinear polynomial space on $T_{h}$ in this paper. The goal is to find $\mathbf{a}_{h}$ so that the following Galerkin formula holds:

$$
\iint_{\Omega} \rho \mathbf{a}_{h} v_{h} \mathrm{~d} s=\iint_{\Omega}\left(p \nabla v_{h}+\rho \mathbf{g} v_{h}\right) \mathrm{d} s-\int_{\partial \Omega} p v_{h} \mathbf{n} \mathrm{d} l, \forall v_{h} \in V_{h},
$$

where $\partial \Omega$ denotes the boundary of $\Omega$, and $\mathbf{n}$ the normal unit vector defined as pointing outward. This equation is then be solved by taking the numerical solution $\mathbf{a}_{h}=\sum_{j=1}^{N} \mathbf{a}^{(j)} \phi_{j}$ and let the test function $v_{h}=\phi_{i}$, where the superscript $(j)$ indicates the nodal value of node $j$ and the subscript $i$ means the bilinear basis function for node $i$. Then, Eq. 5 can be written as the following linear system

$$
\left\{\begin{array}{l}
\mathbf{M A}_{r}=\mathbf{F}_{r} \\
\mathbf{M A}_{z}=\mathbf{F}_{z}
\end{array}\right.
$$

where $\mathbf{M}$ is the mass matrix with its element $M_{i j}=\iint_{\Omega} \rho \phi_{i} \phi_{j} \mathrm{~d} s, \mathbf{A}_{r}$ and $\mathbf{A}_{z}$ are the nodal acceleration vectors at the $r$ and $z$ directions, and $\mathbf{F}_{r}$ and $\mathbf{F}_{z}$ are the corresponding load vectors relating the nodal force. Then, the nodal velocity $\mathbf{u}$ is updated with the explicit integral scheme, and the mesh advances with the fluid material. The material density and the specific internal energy $e_{i n}$ are updated with the Eq.1 and Eq.3.

Then, we need to implement the Eulerian phase in which the deformed mesh is moved back with the material fixed at the advanced position. During this phase, the relative movement between the mesh and the fluid material can be easily obtained, and the convected volume is integrated over the shared edges between adjacent elements. Then, the element-centered variables, $\rho$ and $e_{i n}$, are convected with the monotone upwind scheme for conservation laws (MUSCL) (Benson, 1992; Leer, 1977), which is based on

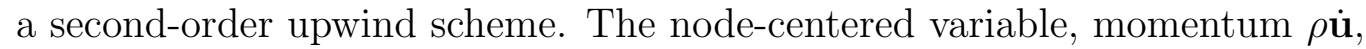
is convected with the half index shift scheme proposed by Benson (2008). These techniques will not be repeated in this paper.

After both the Lagrangian and the Eulerian phases, the pressure $p$ of each fluid phase is updated with the corresponding Equation of State (EoS). In this paper, the water is modeled with the Tammann equation (Ivings et al., 
1998; Liu et al., 2019)

$$
p=\rho e_{i n}(\gamma-1)-\gamma P_{w},
$$

where $\gamma=7.15$ is the specific heat ratio and $P_{w}=330.9 \mathrm{MPa}$ is the reference pressure. As the previous works did (Lee et al., 1968; Liu et al., 2018b; Dobratz, 1981), the internal explosion products are applied the Jones-WilkensLee (JWL) equation as

$$
p=A\left(1-\frac{\omega}{R_{1}} \beta\right) \mathrm{e}^{-\frac{R_{1}}{\beta}}+B\left(1-\frac{\omega}{R_{2}} \beta\right) \mathrm{e}^{-\frac{R_{2}}{\beta}}+\omega \rho e_{i n} .
$$

Herein, $\omega, A, B, R_{1}$, and $R_{2}$ are the material constants as shown in Table $1, \beta=\frac{\rho}{\rho_{0}}$ is the relative density and $\rho_{0}$ is the mass density of the explosive. In this paper, the explosive is taken as trinitrotoluene (TNT) whose material properties are listed in Table 1 , where $e_{i n 0}$ is the initial specific internal energy.

Table 1: Material properties of TNT (Dobratz, 1981).

\begin{tabular}{ccccccc}
\hline$\rho_{0}\left(\mathrm{~kg} / \mathrm{m}^{3}\right)$ & $\omega$ & $A(\mathrm{GPa})$ & $B(\mathrm{GPa})$ & $R_{1}$ & $R_{2}$ & $e_{i n 0}(\mathrm{MJ})$ \\
\hline 1630 & 0.3 & 371.2 & 3.231 & 4.15 & 0.95 & 4.29 \\
\hline
\end{tabular}

\subsection{Pressure balance of mixture in double-phase elements}

One difficulty of the VOF method in dealing with compressible multiphase fluid is how to determine the pressure of the mixture in mixed elements. According to the continuity assumption in FEM, the two fluids at different sides of the interface within an element should share the same pressure. However, after the advance in one-time increment, the changes of the two pressures usually differ from each other because of the difference between their EoS parameters or even the EoSs. Therefore, the balanced pressure must be calculated inside the element. Coralic and Colonius (2014) proposed an analytical mixture rules to calculate the average pressure for the element consisting of fluids described by the Tammann equation. However, it is non-trivial to expand the rules to arbitrary EoSs.

In this paper, an iterative method is proposed for the pressure balance of an element consisting of fluids with Tammann and JWL EoSs. Assume that a concerned element consists of 2 different fluids with their volume fractions denoted by $f_{i}$, where $i$ is the number of the liquid. Then, the interface between 
the two fluids is supposed to move from the side with the higher pressure to the other hand, which changes the fluid fractions and the pressures. This procedure should be accomplished in a short time, which is proportional to the element size. Thus, when the element size is small enough, it can be treated as adiabatic. Denoting the change of the $i_{t h}$ fluid fraction by $\delta f_{i}$, it can be calculated by solving the following nonlinear equation

$$
P_{a d b 1}\left(p_{1}, \frac{f_{1}}{f_{1}+\delta f_{1}}\right)=P_{a d b 2}\left(p_{2}, \frac{f_{2}}{f_{2}-\delta f_{2}}\right),
$$

where $\delta f=\delta f_{1}=\delta f_{2}$, and $P_{a d b}(p, \beta)$ is the adiabatic EoS of the fluid $i$ with respect to the pressure $p_{i}$ before the pressure balance procedure and the density ratio $\beta$. For the Tammann EoS used in this paper, it can be expressed as

$$
P_{a d b}=\left(p+\gamma P_{w}\right) \beta^{\gamma}-\gamma P_{w} .
$$

Similarly, the adiabatic JWL EoS reads (Lee et al., 1968; Liu et al., 2018b; Dobratz, 1981)

$$
P_{a d b}=A \mathrm{e}^{-\frac{R_{1}}{\beta}}+B \mathrm{e}^{-\frac{R_{2}}{\beta}}+C \beta^{1+\omega},
$$

where $C$ is the last term in Eq.8.

The following iteration method is applied to solve Eq.9 by assuming the pressure change is linearly related to the density change with the slope of $C_{i}^{2}$ during each iteration increment, and $i$ is the number of the fluid:

$$
\delta f^{(n)}=\left[\left(p_{1}-p_{2}\right) \frac{f_{1}\left(1-f_{1}\right)}{f_{1}\left(\rho_{1} C_{1}^{2}-\rho_{2} C_{2}^{2}\right)+\rho_{2} C_{2}^{2}}\right]^{(n)},
$$

where $n$ is the number of the iteration step. Then, the volume fractions of the 2 fluids can be updated with

$$
\begin{aligned}
& f_{1}^{(n+1)}=f_{1}^{(n)}+\delta f^{(n)}, \\
& f_{2}^{(n+1)}=f_{2}^{(n)}-\delta f^{(n)} .
\end{aligned}
$$

To maintain the stability of the iteration, $\delta f$ is limited by the following conditions

$$
\delta f^{(n)} \leq S_{c 1} \min \left(f_{1}, f_{2}\right)^{(n)},
$$

where $S_{c 1}$ is a constant fraction which is taken as 0.1 in this paper.

The linear assumption is only used in Eq.12 to estimate the fluid fraction change in each iteration, and then the EoSs of Eq.7 and Eq.8 are used to 
update the new pressure of each phase after the fluid fraction is updated for each fluid in the element. The densities are recalculated with the conservation of mass, and the new pressures are recalculated with the non-linear EoSs. The iteration is stopped when the following criterion is satisfied:

$$
\left|p_{1}-p_{2}\right|^{(n)} \leq S_{c 2} \min \left(p_{1}, p_{2}, P_{\infty}\right)^{(n)},
$$

where $S_{c 2}$ is taken as 0.01 in this paper, and $P_{\infty}$ denotes the undisturbed pressure at the bubble center.

Note that the energy is not conservative in this procedure because the internal energy changes of the two liquids are not equal:

$$
\int_{f_{1}^{(n)}}^{f_{1}^{(n+1)}} p_{1} \mathrm{~d} f+\int_{f_{2}^{(n)}}^{f_{2}^{(n+1)}} \quad p_{2} \mathrm{~d} f \leq 0,
$$

which means that some energy is lost. However, the lost energy must converge to 0 while the element size is decreasing.

\section{Results and Discussion}

\subsection{Model validation and general features of collapsing bubble near solid wall}

We carried an experiment to validate the numerical model, with the bubble generated by underwater discharge in a water tank sized by $0.5 \times 0.5 \times$ $0.5 \mathrm{~m}^{3}$. Though there are significant differences between explosion and spark generated bubbles, such as the bubble formation process, internal gaseous contents, and spatial scales, the underwater discharge experiment has been widely used as an substitution to study underwater explosion bubble dynamics(Jayaprakash et al., 2012; Buogo and Cannelli, 2002; Zhang et al., 2015). The reason is that the two kinds of bubbles follow the same mechanism and have the same motion features during their collapsing phases which are driven by the balance of pressure difference and fluid inertia. The evolution of the bubble is recorded by a high-speed camera. The size of the spark generated bubble is so small that it is not easy to measure the pressure history at a specific point for numerical validation. Alternatively, it can be referred to Liu et al. (2018b) with an underwater explosion experiment in which the pressure history is compared with experimental results. The detailed experiment setup can refer to Zhang et al. (2016). The equivalent maximum bubble radius is $R_{m}=12.08 \mathrm{~mm}$ (calculated with the maximum 
volume). The bubble is initially generated $0.2 \mathrm{~m}$ below the free surface with a horizontal still plate locating $10.78 \mathrm{~mm}$ beneath the bubble. According to the non-dimensionalization theory of bubble dynamics (Zhang and Liu, 2015; Klaseboer et al., 2005a), the gravity is negligible as a result of the small buoyancy parameter $\delta^{2}=\frac{R_{m} \rho g}{P_{\infty}}$. In this case, the internal gas is modeled with the ideal gas equation (Tian et al., 2018)

$$
p=\rho e_{i n}(\gamma-1),
$$

with $\gamma=1.4$ rather than the JWL EoS, because it is not generated by the explosion. The initial internal pressure $p_{0}=100 P_{a t m}$ (Klaseboer and Khoo, 2004; Turangan et al., 2006), where $P_{a t m}=1.01 \times 10^{5} \mathrm{~Pa}$ is the atmosphere pressure.

The computational domain is set as a rectangle size by $4 R_{m}$ and $6 R_{m}$ for the radial and vertical directions, respectively. The element size is taken as $l=0.005 R_{m}, 0.01 R_{m}, 0.02 R_{m}, 0.04 R_{m}$ and $0.06 R_{m}$ to test the convergence of the numerical model. OpenMP parallel technique is used to accelerate the simulation, which takes 4 hours to simulate the case with the finest mesh (the total element number is 240000) on a computer with 16GB memory and Intel i7-8700 $\mathrm{CPU}(3.2 \mathrm{GHz} \times 6)$.

The most crucial difference between the experiment and the numerical model is that the bubble is generated by a continuously discharge, which leads to a much slower expansion phase rather than an instantaneous detonation. But when the discharge is finished, the numerical model can simulate the experiment well. Thus, both the results from the experiment and the numerical simulation are presented from the bubble reaching its maximum volume and the moment is marked as $t=0.0 \mathrm{~ms}(2.27 \mathrm{~ms}$ from the discharge in the experiment and $1.65 \mathrm{~ms}$ from the beginning of the simulation, respectively).

The overall convergence of the numerical model is evaluated in Fig.2 with the volume histories of the bubble with different mesh sizes. As we can see, the pulsation period of the bubble decreases and convergences together with the refining of the computational mesh. When $l$ further decreases from $0.02 R_{m}$ to $0.005 R_{m}$, the improvement of the results is quite limited. In Fig.3 , the bubble profiles at $t=1.58 \mathrm{~ms}$ with different mesh sizes are compared for the convergence test. The coarsest meshes with $l=0.04 R_{m}$ and $l=0.06 R_{m}$ fail in predicting the bubble volume because of their inaccuracy in the bubble pulsating period simulation as shown in Fig.2. When $l$ is reduced to $0.02 R_{m}$, the overall shape of the bubble is well simulated, while the resolution of the 


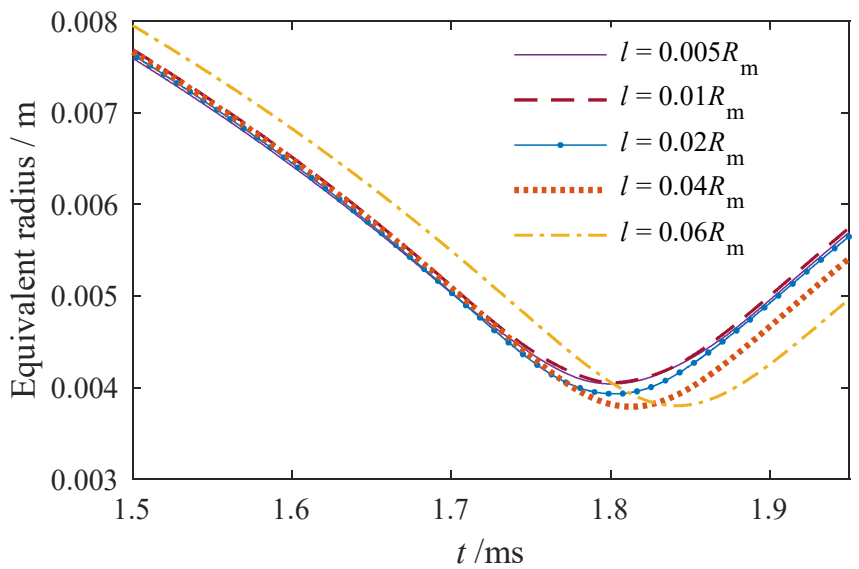

Figure 2: Comparison of equivalent radius histories simulated with different mesh sizes $l=0.005 R_{m}, 0.01 R_{m}, 0.02 R_{m}, 0.04 R_{m}$ and $0.06 R_{m}$, respectively.

mesh is too low to capture the annular jet. Though these evolution details after the jet penetration are not strongly related to the impact load if the jet penetration process is well simulated, the mesh size is taken as $0.01 R_{m}$ to archive better results.

In Fig.4, we compare the experiment (left) and the simulation (right) results of the bubble contraction phase when the bubble is set near a solid wall. The two sets of results agree with each other well in the whole collapsing phase. The wall obviously attracts the bubble. Due to the high pressure above the bubble, there is a high-speed liquid jet forming and impacting on the wall at $t=1.60 \mathrm{~ms}$. The jet speed is up to $71 \mathrm{~m} / \mathrm{s}$ before the impact, and the impact pressure is as high as $4.75 \mathrm{MPa}$ (see the first pressure peak in Fig. 5 in which the hydrodynamic pressure at the wall center is drawn in Fig. 5 with the equivalent radius history and the 3 round marks indicate the moments of the last three subfigures in Fig.4). After the jet impact, the pressure dramatically attenuates. When $t=1.80 \mathrm{~ms}$, the first bubble period finishes, and the local pressure reaches the second peak which is comparable with the previous one caused by the jet impact. This pressure peak corresponds to the bubble pulsating load. In Fig.5, we can see these two pressure peaks occur closely, and the interval is as small as $0.2 \mathrm{~ms}$. In other words, They can enhance the effects of each other and pose the main threats of a collapsing bubble to the nearby structures. 


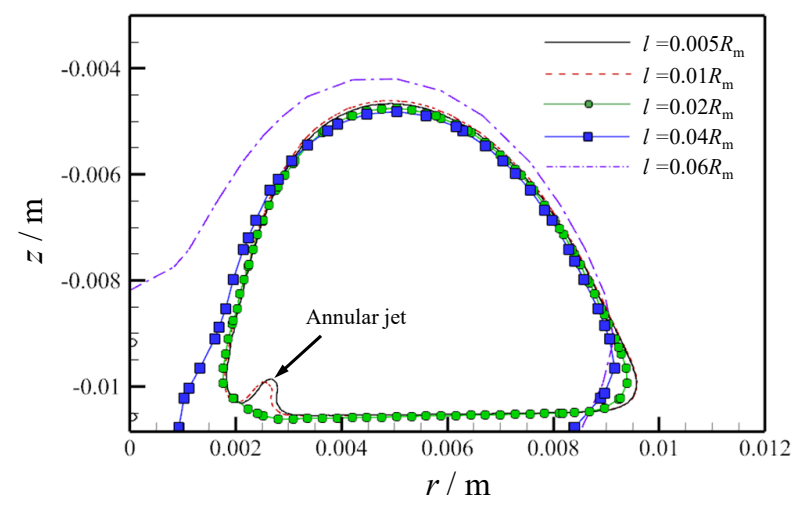

Figure 3: Comparison of simulated bubble profiles at $t=1.58 \mathrm{~ms}$ with different mesh sizes $l=0.005 R_{m}, 0.01 R_{m}, 0.02 R_{m}, 0.04 R_{m}$ and $0.06 R_{m}$, respectively.

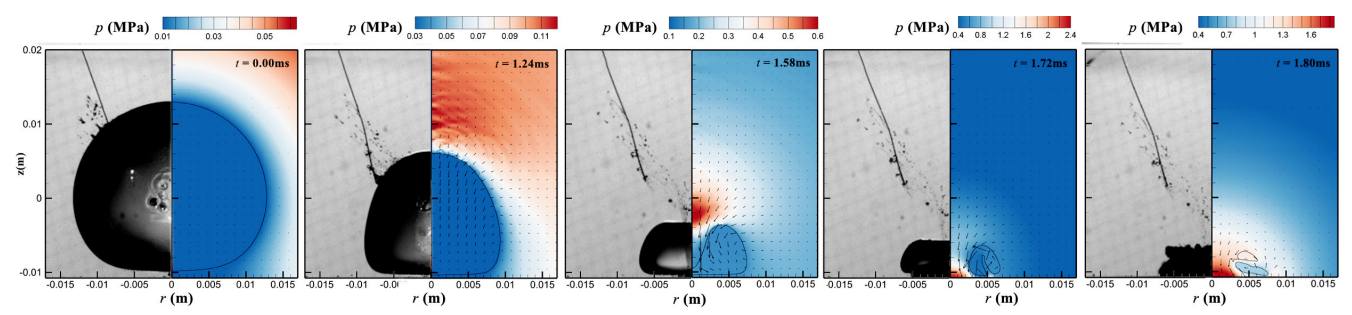

Figure 4: Comparison of results from the experiment (left) and the numerical simulation (right) for a discharge bubble collapsing near a solid wall. The moment when the bubble starts collapsing is set to $t=0 \mathrm{~ms}$. The color contour represents the pressure, and the arrows indicate the fluid velocity.

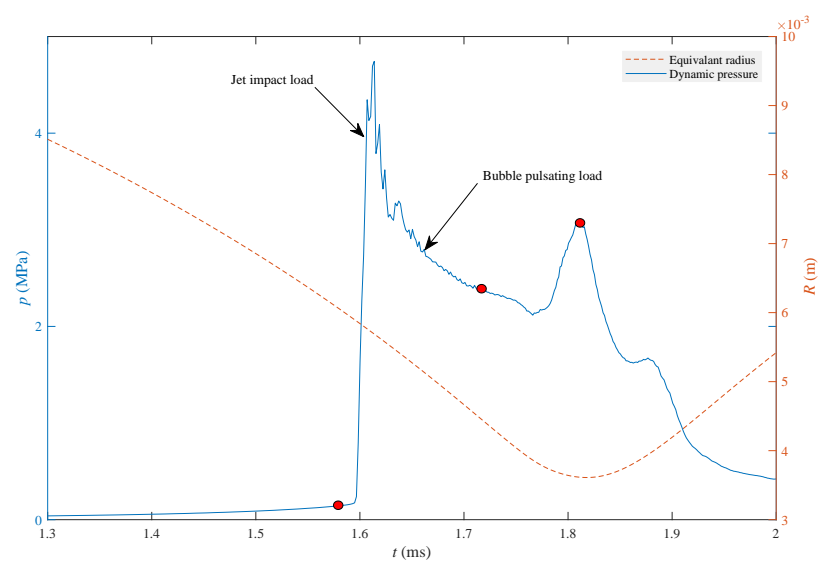

Figure 5: Equivalent bubble radius and the dynamic pressure history of the wall center during the bubble collapsing phase. 


\subsection{Underwater explosion bubble above a horizontal solid wall}

Usually, the gravity of the surrounding fluid generates a downward pressure gradient around the bubble. Because the density of internal gas is much smaller than the surrounding fluid, the gravity-related pressure gradient inside the bubble is negligible such that buoyancy emerges, and the lower part of the bubble collapse faster. As a result, the bubble starts to collapse nonspherically and develop an upward jet in a free-field flow. Then, the solid wall induces the nearby bubble to develop a liquid jet toward the wall. Thus, when these two effects are in the opposite directions, the joint force is expected to be offset by each other, and the greater one dominates the bubble dynamics.

Firstly, we present the simulation of a case of this kind problem, i.e., the solid wall locates right beneath the bubble. Here, we consider the cases that the bubble impacts on a downside solid wall. A TNT explosive weighted $100 \mathrm{~kg}$ is initially detonated at a depth of $50 \mathrm{~m}$. If the explosion occurs in free-field, the maximum radius of the bubble is predicted with the empirical formula presented by Cole (1948):

$$
R_{m}=3.38\left(\frac{W}{h+10.3}\right)^{\frac{1}{3}}
$$

which is $4 \mathrm{~m}$ in this case. Herein, $W$ is the explosive weight and $h$ is the depth from the free surface. Thus, the initial stand-off distance can be non-

dimensionalized to be the distance parameter $\gamma_{d}=\frac{d}{R_{m}}$. At first, the solid horizontal wall is placed beneath the explosive with a distance of $2 \mathrm{~m}$, i.e., $\gamma_{d}=0.5$.

As described in Sect.2.1, the fluid around the bubble interface formulates a Riemann problem initially. A shock wave is emitted and propagates outward in the water, which refers to the shock wave stage. The wave propagates towards the wall and is reflected. Then the reflected shock wave meets the expanding bubble and is reflected again to generate a rarefaction wave towards the wall, as shown in the first subfigure in Fig.6. These waves are reflected by the wall and the bubble interface for several times until the energy dissipates into the surrounding flow, which forms a complex wave structure.

In the rest subfigures of Fig.6, the evolution of the bubble and the flow field pressure are presented. At the moment the bubble stops its expansion (the second subfigure), the downside surface fuses with the solid wall 

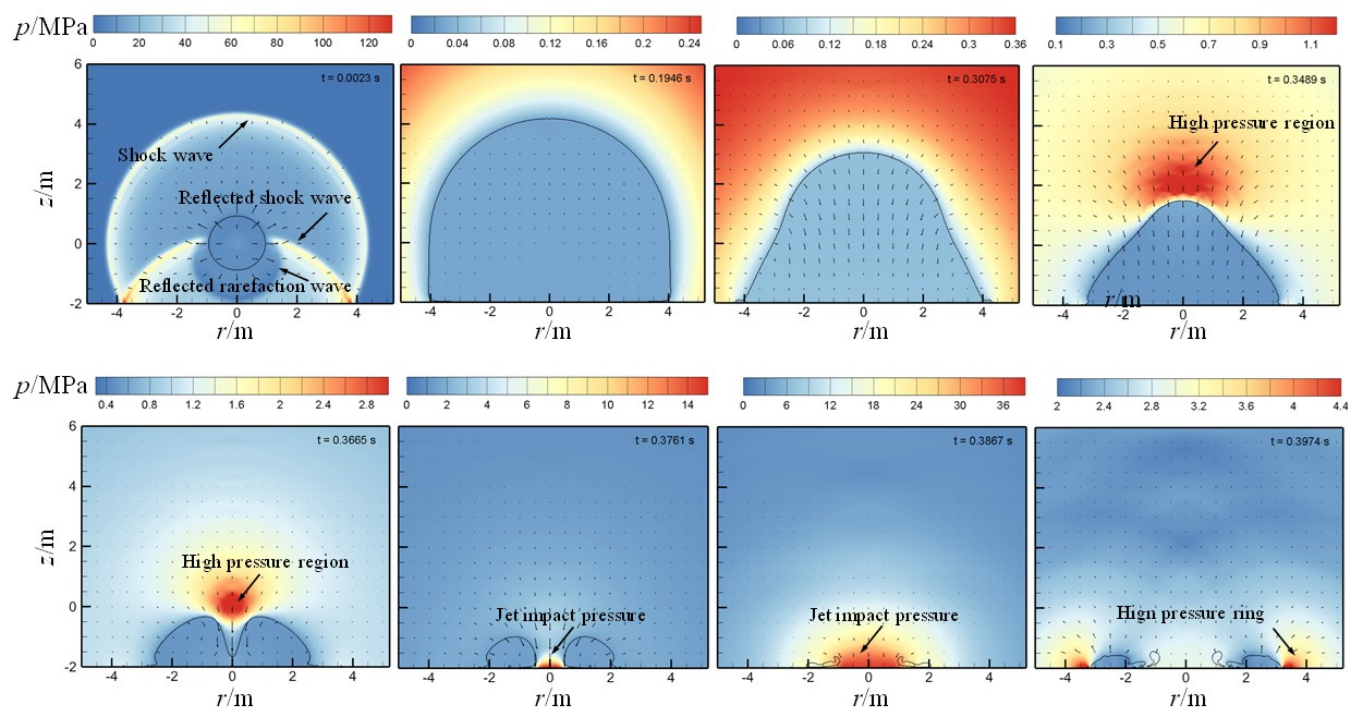

Figure 6: Evolution of the underwater explosion bubble near the downside solid wall generated by an explosive weighted $100 \mathrm{~kg}$ and detonated at a depth of $50 \mathrm{~m}$. The nondimensional stand-off distance of the solid wall is 0.5 . The black curve, color contour, and vector field represent the bubble interface, pressure, and velocity fields, respectively.

together. During the bubble contraction phase, it is attracted by the wall intensively. A high-pressure region emerges above the bubble and pushes the bubble to develop a high-speed downward jet to impact the solid wall straightly. The jet speed is up to $200 \mathrm{~m} / \mathrm{s}$ at the instant of impact and generates a peak pressure of $15 \mathrm{MPa}$. The toroidal bubble continues collapsing until $t=0.3867 \mathrm{~s}$. Meanwhile, the bubble pulsation pressure caused by the internal pressure emerges. Subsequently, the bubble starts rebounding and expands faster in the radial direction, which creates a high-pressure ring around the toroidal bubble close to the wall.

Fig. 7 shows the evolution of the pressure distribution on the wall changing with time. The incident and reflected shock wave overlay together and form a narrow, high-pressure strip in the $R$ - $t$ space. Because of the curvature of the incident shock wave, the slope of the pressure strip gets higher with the increase of time. After $0.0015 \mathrm{~s}$ from the wave reaching the wall, the rarefaction wave reflected by the bubble surface acts on the wall. Thus, a minor suction effect affects the wall right after the impact of the shock wave. Fig.7(b) shows the pressure distribution during the period from $0.36 \mathrm{~s}$ to 0.43 when the jet impact occurs. The jet impact region is very localized. 


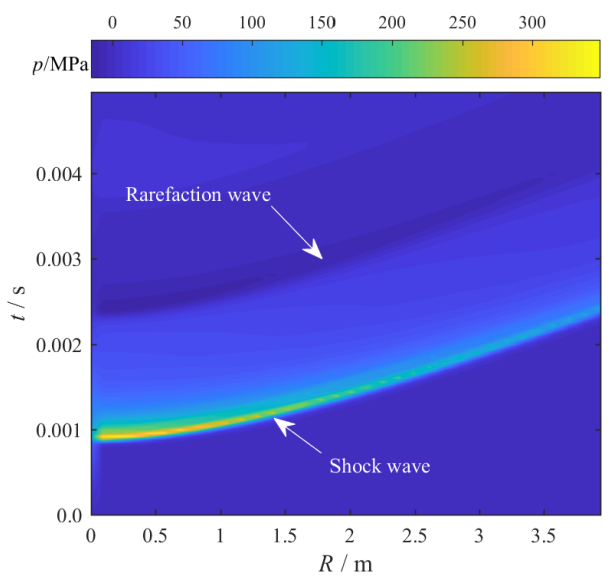

(a) Shock wave phase

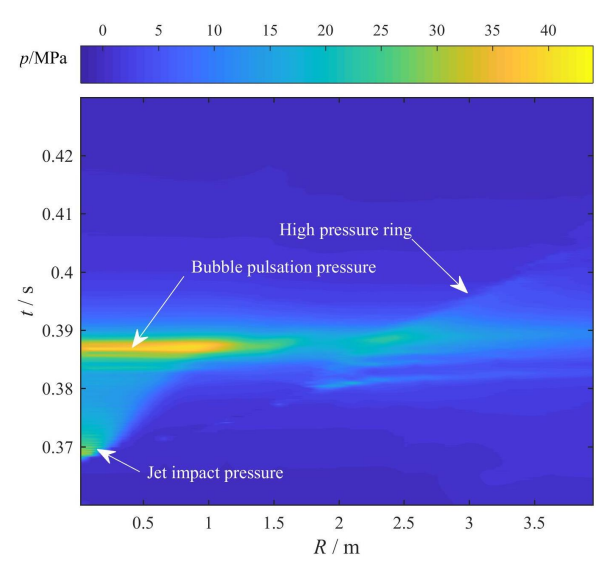

(b) Bubble impact phase

Figure 7: Change of pressure distribution of the solid wall along with time for the same case in Fig.6.

Although the pressure peak is much smaller than the shock wave, about only $30 \mathrm{MPa}$, the duration is much longer. Thus, the total impulse is in the same order. Following the jet impact pressure, the pulsation pressure is emitted from the bubble around $t=0.387 \mathrm{~s}$ when the bubble stops collapsing and starts rebounding. The pressure peak is about $45 \mathrm{MPa}$ and acts in a broader region than the jet impact.

Next, a case with a greater stand-off distance of $4 \mathrm{~m}$ is simulated with the other case parameters remain the same. The bubble evolution is shown in Fig.8. Similar features can be found in this case, such as the multi-reflection of the shock wave between the solid wall and the bubble surface, the highpressure region occurs above the bubble because of the rigid wall effects and the downward liquid jet impacting on the solid wall. However, the jet is thinner but faster. The jet speed is up to $400 \mathrm{~m} / \mathrm{s}$ so that the impact generates a pressure peak of $110 \mathrm{MPa}$, which is comparable to the shock wave, as shown in Fig.9. The thickness of the downward jet is not enlarged as quickly as that of the previous case. Thus the jet rushing pressure region is still very localized even after the pulsating load.

The case with a further greater stand-off distance of $6.2 \mathrm{~m}\left(\gamma_{d}=1.5\right)$ is calculated, and the bubble evolution is shown in Fig.10. Compared with the solid wall, the buoyancy plays a dominant role in the joint effects. The liquid jet in the collapsing phase points straight up, and there is no direct impact 

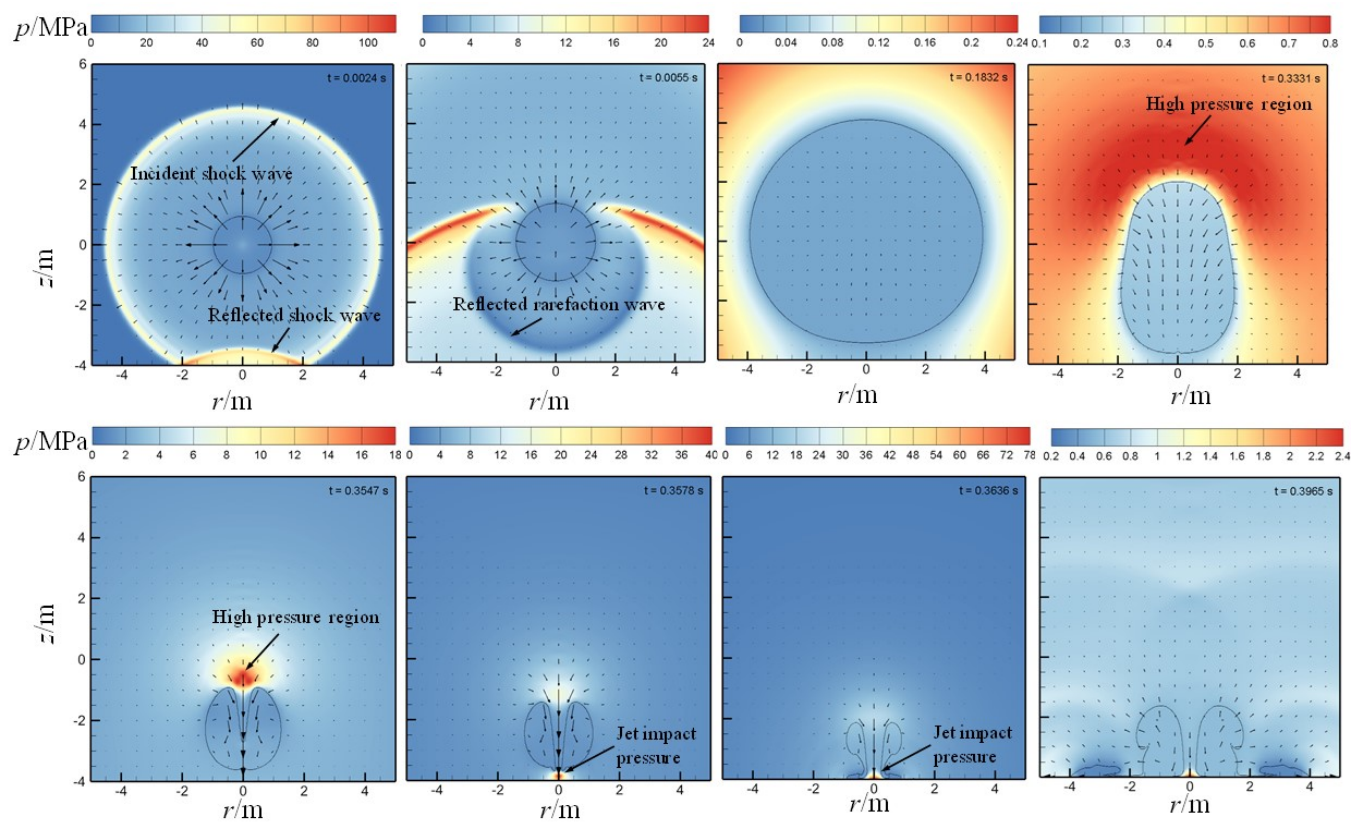

Figure 8: Evolution of the underwater explosion bubble near the downside solid wall generated by an explosive with the same initial conditions as the case in Fig.6 except for the non-dimensional stand-off distance of the wall is 1.0.

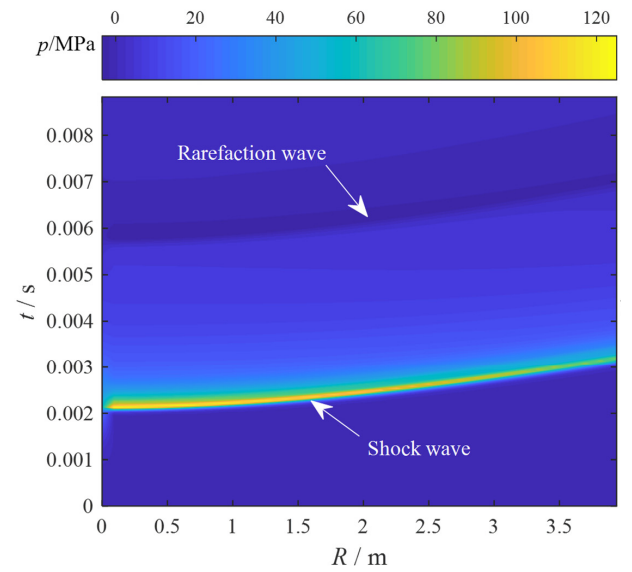

(a) Shock wave phase

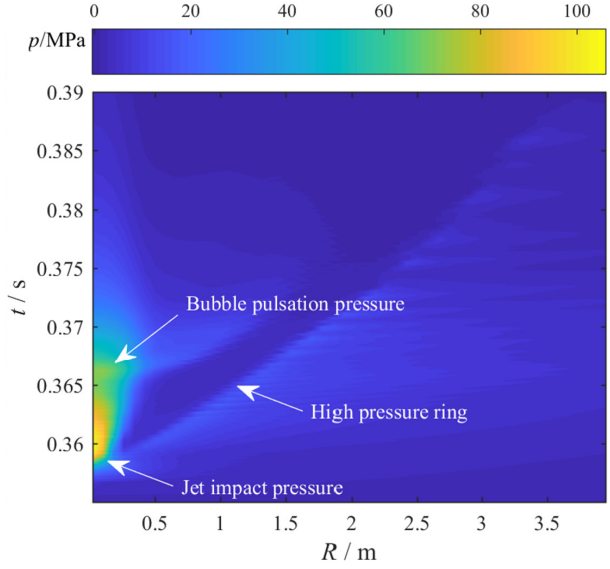

(b) Bubble impact phase

Figure 9: Change of pressure distribution of the solid wall along with time for the same case in Fig.8. 

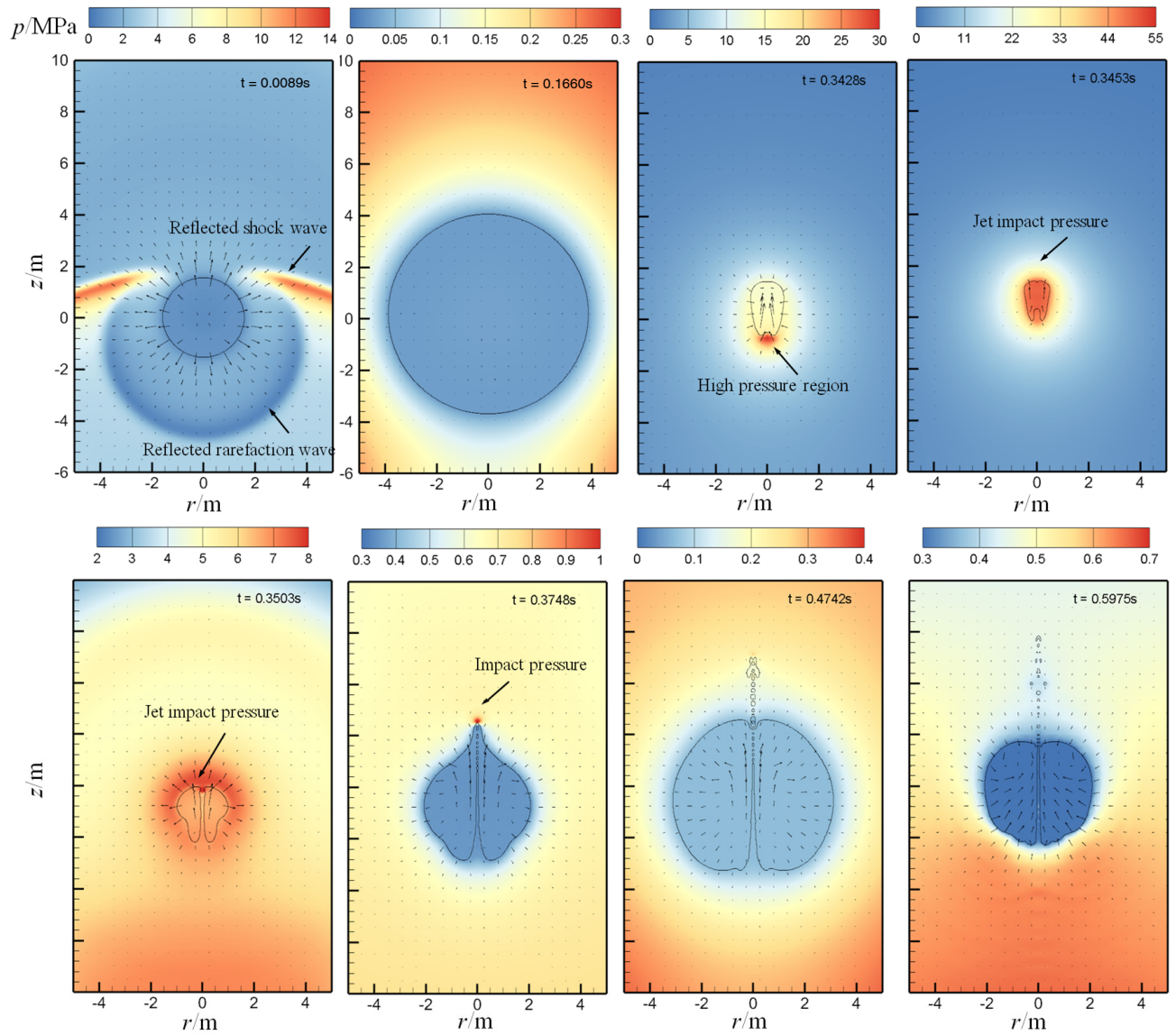

Figure 10: Evolution of the underwater explosion bubble near the downside solid wall generated by an explosive with the same initial conditions as the case in Fig.6 except for the non-dimensional stand-off distance of the wall is 1.5.

on the wall. Significantly, the bubble is penetrated by the jet at $t=0.3428 \mathrm{~s}$ during its rebound because the most effects of the wall and buoyancy offset each other, and their conjunct effect is quite weak. The upside bubble splits into many small bubbles moving upward instantly at the end of the expanding phase in the second pulsating cycle. Then, the central bubble begins to collapse, and a high-pressure region develops under it, which indicates the effects of the wall is also much weaker than that of buoyancy in this pulsating cycle. As shown in Fig.11, the pressure peak magnitude has been greatly weakened whether in the shock wave phase or the bubble impact phase. No- 


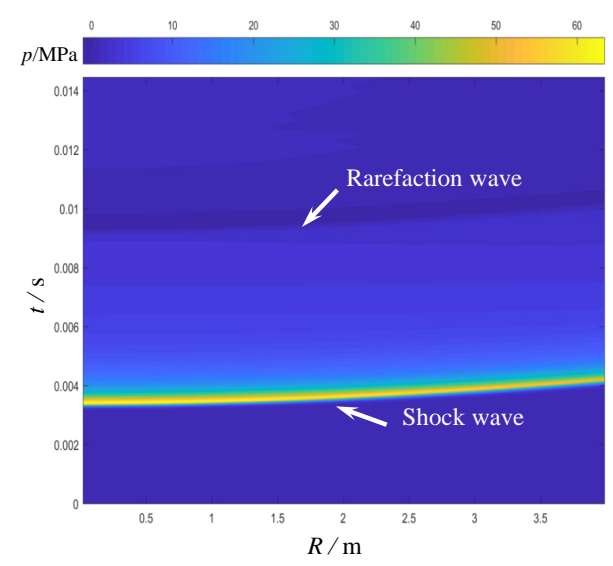

(a) Shock wave phase

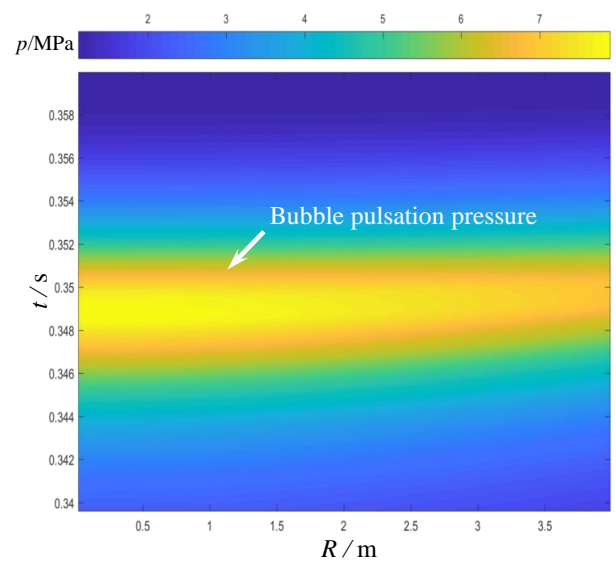

(b) Bubble impact phase

Figure 11: Change of pressure distribution of the solid wall along with time for the same case in Fig.10.

tice that the explosion loads effects on the wall is getting closer to the plane forces, and even the entire wall is affected by the bubble pulsation pressure.

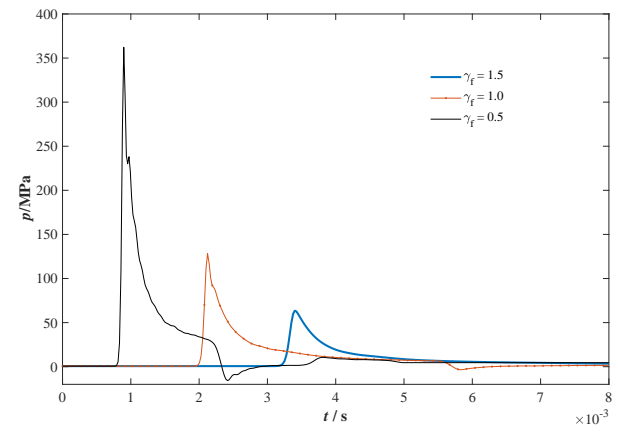

(a) Shock wave phase

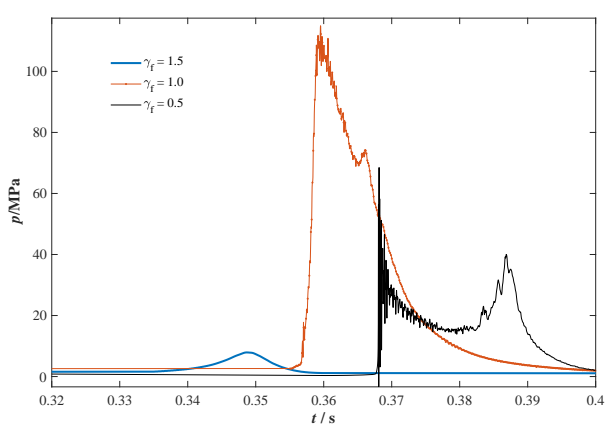

(b) Bubble impact phase

Figure 12: Comparison of pressure histories at the center of the solid wall of the 3 cases with $\gamma_{d}=0.5,1.0$, and 1.5 as shown in Fig.6, Fig.8, and Fig.10, respectively.

Fig.12 shows the comparison of the impact pressure histories for these 3 cases at the center of the solid wall where $r=0.0 \mathrm{~m}$. In the shock wave phase, the pressure peak delays and decays along with the increase of $\gamma_{d}$. Because of the absence of the cavitation model in the present numerical model, negative pressure appears when the rarefaction wave reflected by the bubble reaches 
the stable wall. As for the bubble impact phase, we can see that the bubble load is not monotonic with the stand-off distance. The jet impact pressure and the bubble pulsation pressure reach their maximums when $\gamma_{d}=1.0$. In the case with $\gamma_{d}=0.5$ which is the smallest in the 3 cases, the liquid jet starts developing at an earlier time dominated by the solid wall such that the jet is thick but slow, which leads to a smaller jet impact pressure. During the bubble collapsing, a more substantial part of total energy is occupied by the surrounding flow because of the non-spherical movement. Thus, the bubble pulsation pressure is also weakened. As for the case with $\gamma_{d}=1.5$, the wall is so far that the bubble is dominated by buoyancy and no jet impacts on the wall. The pulsation pressure, in this case, is even smaller than the case with $\gamma_{d}=0.5$ as a result of the further distance. At last, the case with a medium $\gamma_{d}=1.0$ takes all the positive factors to enhance the jet impact and pulsation loads. Thus, in the view of the bubble impact load, the impact pressure is not monotonic to the stand-off distance, and the most severe threaten is not generated by the closest underwater explosion.

As stated before, the jet direction is determined by the conjunct effect of buoyancy and solid wall. Buoyancy does not change with different distances because it only depends on the pulsation period, gravity, bubble size, and density difference across the bubble interface. According to the mirror theory, the effect of the solid wall can be imitated by an imaginary bubble on the other side. The pressure generated by the imaginary bubble is proportional to $1 / d$. Thus, the effect of the solid wall on the oscillating bubble should be comparable to $1 / d^{2}$. When distance $d$ is small, the solid wall effect is greater than that of buoyancy so that the jet points to the solid wall. When $d$ is great enough, the jet will turn its direction to that of buoyancy because the solid wall effect is too small. To demonstrate the transition from downward jet to upward jet, the bubble profiles when they are penetrated by the jets with different stand-off distances are plotted together in Fig.13, in which $\gamma_{d}=\infty$ represents that the bubble oscillates in the free field. It can be seen that the transition stand-off distance is about 1.3. When $\gamma_{d} \geq 1.0$, the downward jet can not impact directly on the solid wall. The water layer between the impact point and the wall can act as a cushion to protect the wall. However, the jet speed can be much higher than that with a smaller $\gamma_{d}$. With $\gamma_{d}$ increasing to 1.3, the effects of buoyancy and solid wall are comparable, and the bubble tends to be split into parts by an annular jet. If $\gamma_{d}$ further increases, the liquid jet turns to upward, and the bubble motion tends to approach that in free-field. It should be noted that the transition $\gamma_{d}$ depends 


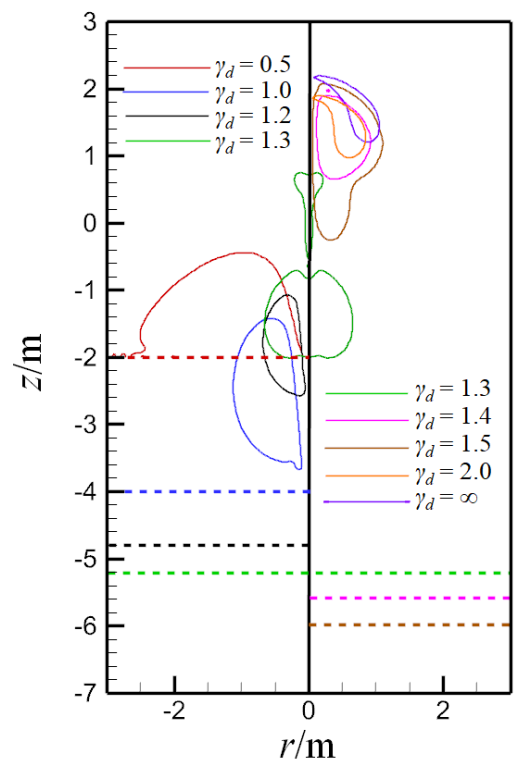

Figure 13: Bubble profiles when they are penetrated by jets with stand-off distance (left) $\gamma_{d}=0.5,1.0,1.2,1.3$ and (right) $\gamma_{d}=1.3,1.4,1.5,2.0, \infty$, respectively. The nondimensional stand-off distance of the solid wall is 0.5. Each dotted line represents the wall position, and its color corresponds to the bubble in the same case. It is noted that when $\gamma_{d} \geq 1.0=\infty$, there is no wall in the free field.

on the buoyancy parameter, i.e., with a different buoyancy parameter, the transition stand-off distance will also change.

\subsection{Underwater explosion bubble beneath a solid horizontal wall}

In this section, the bubble dynamics of an underwater explosion bubble generated above a solid wall is investigated. All the initial conditions are the same as the first case in Sect.3.2 except the solid wall located above the bubble, which indicates that its effects on the bubble are in the same direction as those of buoyancy. In the first stage, i.e., the shock wave propagation stage, the characteristic time is too short for the buoyancy to have a noticeable effect. Thus, similar results are expected to be observed. After that, the contact discontinuity, i.e., the bubble interface, expands quickly and evolves non-spherical motion because of the effects of buoyancy and solid wall. Fig.14 displays the bubble evolution, from which we can see that the bubble is also slightly rejected by the wall before reaching its maximum volume. After $t=0.1775 \mathrm{~s}$, the bubble starts collapsing, and an upward jet develops in the 


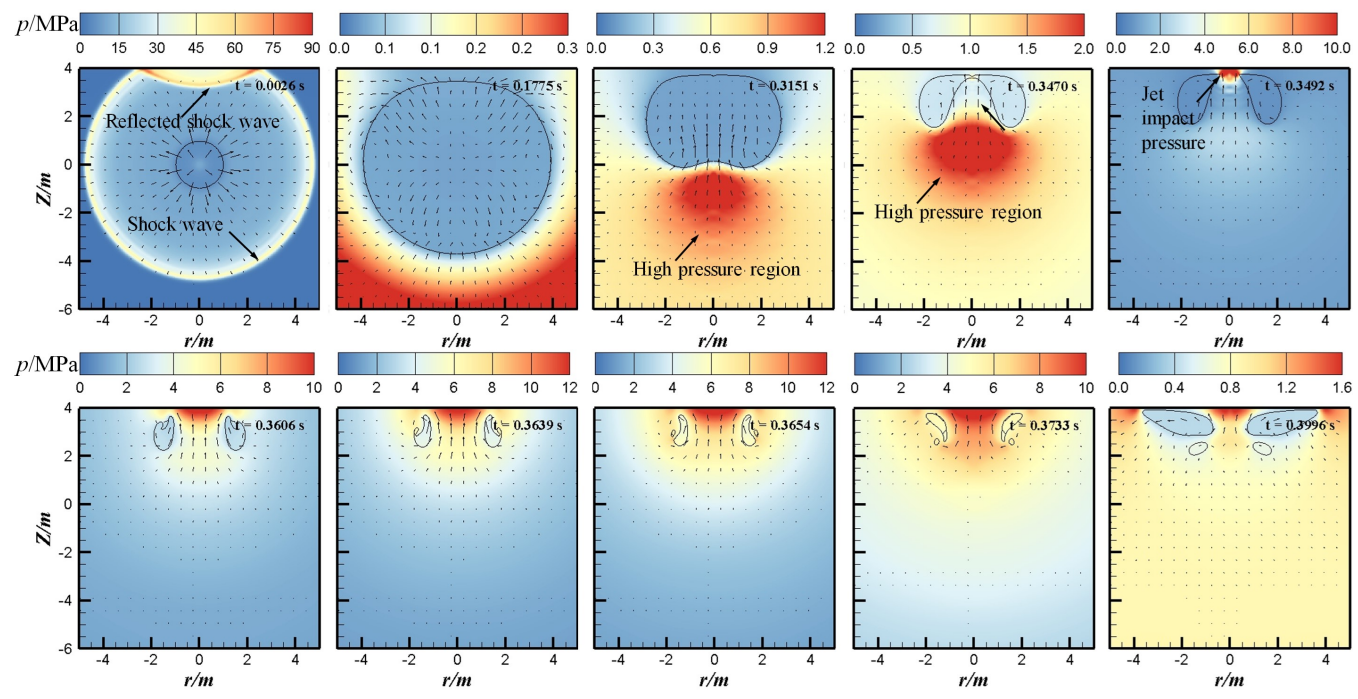

Figure 14: Evolution of the underwater explosion bubble near the upside solid wall where $W=100 \mathrm{~kg}, h=50 \mathrm{~m}$, and the non-dimensional stand-off distance is 1.0 .

joint action of the wall and the buoyancy. Because the two effects are in the same direction, the jet is better developed when it reaches the different bubble interface compared with the counterpart case in the previous section. Thus, the jet is much thicker than that in the counterpart case with a downside solid wall and penetrates the bubble at $t=0.3492 \mathrm{~s}$. As a result, the impact pressure is not as localized as before. Then, the toroidal bubble continues shrinking, and the high-pressure region is expanding because the bubble pulsating pressure is taking over domination. After the collapsing velocity of the bubble decaying to zero, it starts rebounding with the jet current continuing rushing onto the solid wall.

The pressure evolution of the solid wall along with $r$ and $t$ is shown in Fig.15 for shock wave stage and jet impact stage, respectively. There are two noticeable pressure strips in Fig.15(a) in which the earlier one on the lower side is the joint pressure of the shock wave emitted from the bubble and the reflected shock wave by the solid wall. As discussed above, the reflected shock wave is indicated by the expanding bubble surface to generate a rarefaction wave propagating towards the wall, which refers to the later pressure strip in Fig.15(a) on the upper side. We can see that the pressure peak of the wave on the wall decays smoothly along with $r$, which means that the shock wave does impact a broad range of solid wall. On the contrary, the jet 


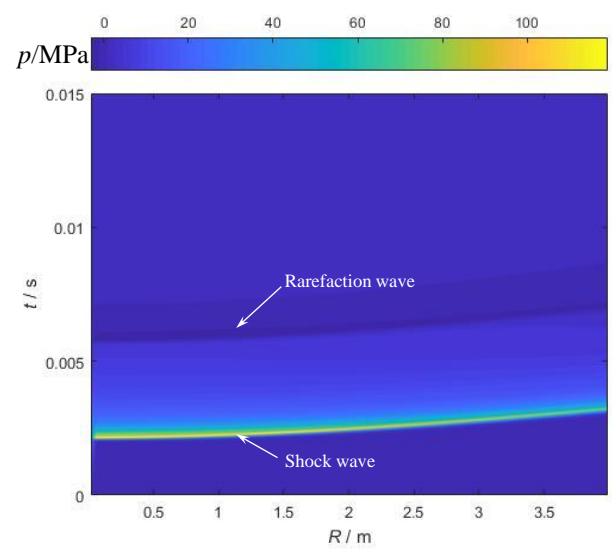

(a) Shock wave phase

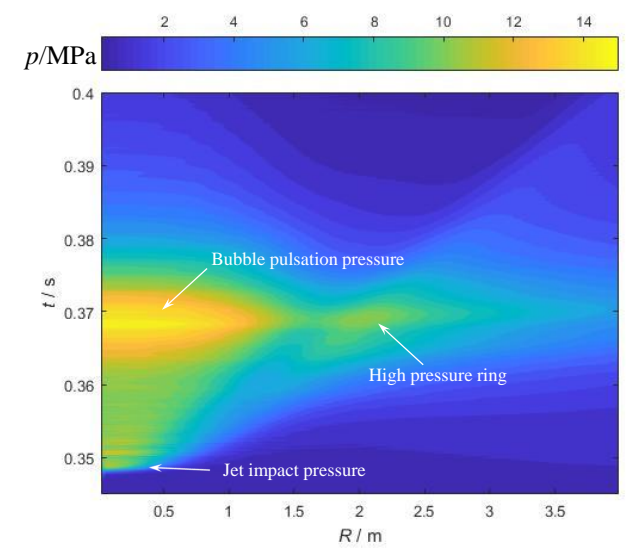

(b) Bubble impact phase

Figure 15: Change of pressure distribution of the solid wall along with time for the same case in Fig.14.

impact load is much more localized within the area of $r<0.5 \mathrm{~m}$, as shown in Fig.15(b). The jet impact pressure wave propagates along with $r$ and forms the diagonal pressure strip. At the end of the first pulsation cycle, when $t=0.37 \mathrm{~s}$, the bubble pulsating pressure comes to its peak. The pulsating pressure is similar to the shock wave with a relatively more extensive impact range. The pressure amplitude is smaller but lasts for a longer time. In the view of the loaded impulse, the bubble load is even more destructive than the shock wave.

At last, the results of a case with a stand-off distance of 1.5 are presented in Fig.16. Because of the greater stand-off distance, the penetration point is still about $2 \mathrm{~m}$ away from the solid wall such that the jet does not impact on the wall directly. Thus, the impact pressure is only about $6 \mathrm{MPa}$ and is much smaller than that of the previous case. Significant differences are observed compared with the case with the same stand-off distance and a downside wall, which is shown in Fig.10. The jet still points to the solid wall, although the stand-off distance is great. The bubble motion will approach that of a underwater explosion in the free field which is dominated by gravity and develops an upward jet penetrating the bubble if the stand-off distance further increases. 

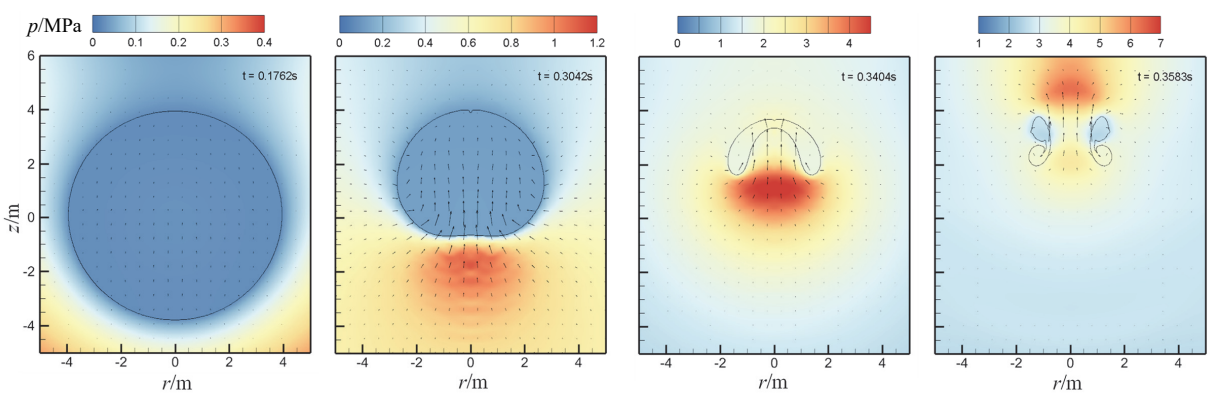

Figure 16: Evolution of the underwater explosion bubble near the upside solid wall where $W=100 \mathrm{~kg}, h=50 \mathrm{~m}$ and the non-dimensional stand-off distance is 1.5 .

\section{Conclusion}

In this paper, the EFEM is introduced to simulate the evolution and impact load of the underwater explosion bubble on a solid wall. An experiment validates the simulation results of the numerical model. Then, several cases of underwater explosion bubbles initiated both above and beneath a solid horizontal wall are simulated. Through the analysis of the results, the following conclusions are drawn.

The jet development of a collapsing bubble is greatly affected by the location of the nearby solid wall. As for the bubble pulsating in the vicinity of a downside wall, the wall and buoyancy compete to gain domination in the direction of the liquid jet. With a small stand-off distance from the wall, the bubble is dominated by its attraction. On the contrary, buoyancy dominates jet development when the stand-off distance is far enough. However, the jet speed and impact pressure reach their peaks at a certain stand-off distance with which jet impact occurs latter and has more time to speed up. As for the upside wall, the bubble dynamics is more straightforward because the two effects of buoyancy and solid wall are in the same direction. With their joint forces, the jet shows earlier and broader when the bubble is penetrated.

Three components of the impact loads of an underwater explosion on a nearby solid wall are investigated through the pressure distribution on the $r-t$ plane. Shock wave tends to be of high pressure but short duration. It affects a broad range of the solid wall and decays smoothly. The jet impact pressure is very localized within the range of impact. Its pressure peak is comparable to the shock wave, but it lasts much longer. As for the bubble pulsation load, it is similar to the shock wave in the distribution but has lower pressure peak and longer duration. The last two loads can be very 
close to each other in the time domain and lead to more destructive effects on nearby structures. With a proper stand-off distance, they can pose severer threaten than the shock wave.

\section{Acknowledgments}

The authors would like to acknowledge the support of the National Natural Science Foundation of China (Grant No. 51879050, 51609044), the China Postdoctoral Science Foundation (Grant No. 2018T110271), the State Scholarship Fund of China (Grant No.201906680004) and the Industrial Technology Development Program (Grant No. JCKY2017604C002).

\section{References}

R. H. Cole. Underwater explosion. Princeton University Press, Princeton USA, 1948.

E. Klaseboer, K. C. Hung, C. W. Wang, and B. C. Khoo. Experimental and numerical investigation of the dynamics of an underwater explosion bubble near a resilient/rigid structure. Journal of Fluid Mechanics, 53(7): 387-413, 2005a.

C. C. Liang and Y. S. Tai. Shock responses of a surface ship subjected to noncontact underwater explosions. Ocean Engineering, 33(5-6):748-772, APR 2006. ISSN 0029-8018. doi: 10.1016/j.oceaneng.2005.03.011.

Y. L. Liu, A. M. Zhang, Z. L. Tian, and S. P. Wang. Numerical investigation on global responses of surface ship subjected to underwater explosion in waves. Ocean Engineering, 161:277-290, 2018a.

Z. L. Tian, Y. L. Liu, S. P. Wang, A. M. Zhang, and Y. W. Kang. Dynamic response of floating body subjected to underwater explosion bubble and generated waves with 2D numerical model. CMES-Computer Modeling in Engineering \& Sciences, 118(2):397-423, 2019. ISSN 1526-1492. doi: 10.31614/cmes.2019.04419.

W. Lai and A. A. Khan. A discontinuous Galerkin method for twodimensional shock wave modeling. Modelling and Simulation in Engineering, 2011:782832, 2011. 
M. A. Sprague and T. L. Geers. A spectral-element method for modelling cavitation in transient fluid-structure interaction. International Journal for Numerical Methods in Engineering, 60(15):2467-2499, 2004.

M. S. Plesset and R. B. Chapman. Collapse of an initially spherical vapour cavity in the neighbourhood of a solid boundary. Journal of Fluid Mechanics, 47(2):283-290, 1970.

M. Lenoir. Numerical calculation of the collapse of a cavitation bubble near a wall or a free surface. Journal De Mecanique, 15(4):687-703, 1976.

M. C. Hooton, J. R. Blake, and W. K. Soh. Behaviour of an underwater explosion bubble near a rigid boundary: Theory and experiment. In J. R. Blake, J. M. Boulton-Stone, and N. H. Thomas, editors, Bubble Dynamics and Interface Phenomena, pages 421-428, Dordrecht, 1994. Springer Netherlands.

S. G. Zhang, J. H. Duncan, and G. L. Chahine. The final stage of the collapse of a cavitation bubble near a rigid wall. Journal of Fluid Mechanics, 257 (257):147-181, 2006.

J. R. Blake and D. C. Gibson. Cavitation bubbles near boundaries. Annual Review of Fluid Mechanics, 19(1):99-123, 1987.

M. Lee, E. Klaseboer, and B. C. Khoo. On the boundary integral method for the rebounding bubble. Journal of Fluid Mechanics, 570(570):407-429, 2007.

P. Cui, A. M. Zhang, S. P. Wang, and B. C. Khoo. Ice breaking by a collapsing bubble. Journal of Fluid Mechanics, 841:287-309, 2018.

S. Li, A. M. Zhang, R. Han, and Q. Ma. 3D full coupling model for strong interaction between a pulsating bubble and a movable sphere. Journal of Computational Physics, 392:713-731, 2019.

Y. L. Zhang, K. S. Yeo, B. C. Khoo, and W. K. Chong. Simulation of three-dimensional bubbles using desingularized boundary integral method. International Journal for Numerical Methods in Fluids, 31(8):1311-1320, 2015. 
Q. X. Wang, K. S. Yeo, B. C. Khoo, and K. Y. Lam. Vortex ring modelling of toroidal bubbles. Theoretical Computational Fluid Dynamics, 19:303-317, 2005 .

C. Wang, B. C. Khoo, and K. S. Yeo. Elastic mesh technique for 3D BIM simulation with an application to underwater explosion bubbles. Computers and Fluids, 32(9):1195-1212, 2003.

A. Dadvand, M. Dawoodian, B. C. Khoo, and R. Esmaily. Spark-generated bubble collapse near or inside a circular aperture and the ensuing vortex ring and droplet formation. Acta Mechanica Sinica, 2013.

Q. X. Wang. Numerical simulation of violent bubble motion. Physics of Fluids, 16(5):1610-1619, 2004.

Q. X. Wang. Non-spherical bubble dynamics of underwater explosions in a compressible fluid. Physics of Fluids, 25(7), 2013.

A. M. Zhang and Y. L. Liu. Improved three-dimensional bubble dynamics model based on boundary element method. Journal of Computational Physics, 294(C):208-223, 2015.

E. Johnsen and T. Colonius. Numerical simulations of non-spherical bubble collapse. Journal of Fluid Mechanics, 629:231-262, 2009.

L. T. Liu, X. L. Yao, A. M. Zhang, and Y. Y. Chen. Numerical analysis of the jet stage of bubble near a solid wall using a front tracking method. Physics of Fluids, 29:012105, 2017.

Z. L. Tian, Y. L. Liu, A. M. Zhang, and S. P. Wang. Analysis of breaking and re-closure of a bubble near a free surface based on the Eulerian finite element method. Computers and Fluids, 170:41-52, 2018.

Y. L. Liu, A. M. Zhang, Z. L. Tian, and S. P. Wang. Investigation of freefield underwater explosion with Eulerian finite element method. Ocean Engineering, 166:182-190, 2018b.

Y. L. Liu, A. M. Zhang, Z. L. Tian, and S. P. Wang. Dynamical behavior of an oscillating bubble initially between two liquids. Physics of Fluids, 31: 092111, 2019. 
S. Anwar. Three-dimensional modeling of coalescence of bubbles using Lattice Boltzmann model. Computers and Fluids, 184:178-186, 2019.

E. Klaseboer, B. C. Khoo, and K. C. Hung. Dynamics of an oscillating bubble near a floating structure. Journal of Fluids and Structures, 10(2):1-10, $2005 b$.

S. R. Wu and L. Gu. Introduction to the explicit finite element method for nonlinear transient dynamics. John Wiley, Hoboken, New Jersey, 2012.

D. J. Benson. Computational methods in Lagrangian and Eulerian hydrocodes. Computer Methods In Applied Mechanics And Engineering, 99: 235-394, 1992.

B. V. Leer. Towards the ultimate conservative difference scheme iv: a new approach to numerical convection. Journal of Computational Physics, 23: 276-299, 1977.

D. J. Benson. Momentum advection on unstructured staggered quadrilateral meshes. International Journal for Numerical Methods in Engineering, 75: 1549-1580, 2008.

M. J. Ivings, D. M. Causon, and E. F. Toro. On Riemann solvers for compressible liquids. International Journal for Numerical Methods in Fluids, 28:395-418, 1998.

E. L. Lee, H. C. Hornig, and J. W. Kury. Adiabatic expansion of high explosive detonation products. Report UCRL-50422, University of California, 1968.

B. M. Dobratz. LLNL explosives handbook: properties of chemeical explosives and explosive simulants. Technical Report UCRL-52997, Lawrence Livermore National Lab., 1981.

V. Coralic and T. Colonius. Finite-volume WENO scheme for viscous compressible multicomponent flows. Journal of Computational Physics, 274: 95-121, 2014.

A. Jayaprakash, C. T. Hsiao, and G. Chahine. Numerical and experimental study of the interaction of a spark-generated bubble and a vertical wall. Journal of Fluids Engineering, 134(3), 03 2012. ISSN 0098-2202. doi: 10.1115/1.4005688. URL https://doi .org/10.1115/1.4005688. 031301. 
S. Buogo and G. B. Cannelli. Implosion of an underwater spark-generated bubble and acoustic energy evaluation using the Rayleigh model. Journal of the Acoustical Society of America, 111(6):2594-2600, JUN 2002. ISSN 0001-4966. doi: 10.1121/1.1476919.

A. M. Zhang, P. Cui, J. Cui, and Q. X. Wang. Experimental study on bubble dynamics subject to buoyancy. Journal of Fluid Mechanics, 776:137-160, AUG 2015. ISSN 0022-1120. doi: 10.1017/jfm.2015.323.

S. Zhang, S. P. Wang, and A. M. Zhang. Experimental study on the interaction between bubble and free surface using a high-voltage spark generator. Physics of Fluids, 28:032109, 2016.

E. Klaseboer and B. C. Khoo. An oscillating bubble near an elastic material. Journal of Applied Physics, 96(10):5808-5818, 2004.

C. K. Turangan, G. P. Ong, E. Klaseboer, and B. C. Khoo. Experimental and numerical study of transient bubble-elastic membrane interaction. Journal of Applied Physics, 100:054910, 2006. 